\title{
Highlights from the ATLAS experiment
}

\author{
Martin Spousta, on behalf of the ATLAS Collaboration \\ Faculty of Mathematics and Physics, Charles University \\ V Holešovičkách 2, Prague 180 00, Czech Republic
}

\begin{abstract}
The ATLAS experiment at the LHC has undertaken an extensive program to probe and characterize the hot and dense quark-gluon plasma created in relativistic heavy ion collisions and, in general, to study non-perturbative and collective properties of the strong interaction. In this report we summarize the most recent results on the collectivity in $p p, p+\mathrm{Pb}$ and nucleus-nucleus collisions, results on jets, quarkonia, heavy flavor and electroweak boson production, measured in $\mathrm{Pb}+\mathrm{Pb}$ and $p+\mathrm{Pb}$ collisions. This report covers also new results on electromagnetic processes studied in ultra-peripheral collisions.
\end{abstract}

Keywords: Heavy-ion collisions, ATLAS, LHC, collectivity in hadronic collisions, azimuthal anisotropies, correlations and fluctuations, ridge, jets, electroweak boson production, quarkonium production, heavy-flavor production, ultra-peripheral collisions, photoproduction, light-by-light scattering

\section{Introduction}

The ATLAS experiment [1] at the LHC has undertaken an extensive program to probe and characterize the hot and dense quark-gluon plasma (QGP) created in relativistic heavy ion collisions and, in general, to study non-perturbative and collective properties of the strong interaction. During LHC Run 2, several runs dedicated to heavy-ion physics were performed. The collision data that are used in studies by ATLAS, along with information on the total integrated luminosity, is summarized in Table 1.

Section 2 of this report summarizes the latest results on the collectivity in $p p, p+\mathrm{Pb}$ and nucleus-nucleus collisions. Section 3 covers results on penetrating probes of QGP in $\mathrm{Pb}+\mathrm{Pb}$ collisions, namely new results on jets and quarkonia suppression. Section 4 summarizes results on physics of initial state and nuclear geometry, namely new results on electroweak boson production in $\mathrm{Pb}+\mathrm{Pb}$ collisions and photon and jet production in $p+\mathrm{Pb}$ collisions. Section 5 covers new results on the light-by-light scattering and the results on the electromagnetic production of muons which may be used also as penetrating probes of QGP. Unless otherwise stated, by $p p, p+\mathrm{Pb}$, and $\mathrm{Pb}+\mathrm{Pb}$ collisions we mean these collisions at the centre-of-mass energy of $5.02 \mathrm{TeV}$ per colliding nucleon pair.

Email address: martin.spousta@mf . cuni.cz (Martin Spousta, on behalf of the ATLAS Collaboration) 


\begin{tabular}{|c|c|c|c|}
\hline System & Year & $\sqrt{s_{\mathrm{NN}}}[\mathbf{T e V}]$ & $\mathbf{L}_{\text {int }}$ \\
\hline$p p$ & $2015+2017$ & 5.02 & $28 \mathrm{pb}^{-1}+0.26 \mathrm{fb}^{-1}$ \\
\hline $\mathrm{Pb}+\mathrm{Pb}$ & $2015+2018$ & 5.02 & $0.49 \mathrm{nb}^{-1}+1.72 \mathrm{nb}^{-1}$ \\
\hline$p+\mathrm{Pb}$ & 2016 & 5.02 & $0.4 \mathrm{nb}^{-1}$ \\
\hline$p+\mathrm{Pb}$ & 2016 & 8.16 & $165 \mathrm{nb}^{-1}$ \\
\hline$p p$ & $2017+2018$ & 13 & $150 \mathrm{pb}^{-1}+193 \mathrm{pb}^{-1}$ \\
\hline $\mathrm{Xe}+\mathrm{Xe}$ & 2017 & 5.44 & $3 \mu \mathrm{b}^{-1}$ \\
\hline
\end{tabular}

Table 1. Summary of LHC Run 2 datasets used by the ATLAS experiment. For each colliding system, the years of data-taking, along with the centre-of-mass energy per colliding nucleon and total integrated luminosity are provided.

\section{Collectivity in $p p, p+\mathrm{Pb}$ and nucleus-nucleus collisions}

Presence of significant azimuthal anisotropy, also known as flow phenomenon, in nucleus-nucleus collisions is one of the most important arguments for the presence of a hot and dense QGP in those collisions. While the azimuthal anisotropies were measured in great details at many energies and over several collision systems both at the LHC and RHIC, there are still open questions left to be addressed: What is the origin of the flow in "small systems" such as $p p$ or $p+\mathrm{Pb}$ ? Is there some universality in the flow phenomenon across collision systems? What is the role of geometry and the initial state and its fluctuations? What is the source of the flow of heavy quarks? What is the origin of the flow at high transverse momenta? In this section we discuss the latest results by ATLAS which may help to find answers to these questions.

The azimuthal anisotropy in nucleus-nucleus collisions was quantified in details by measuring flow harmonics $v_{2}-v_{7}$ and $v_{2}-v_{6}$ in $\mathrm{Pb}+\mathrm{Pb}$ and $\mathrm{Xe}+\mathrm{Xe}$ collisions, respectively [2,3]. Significant $v_{2}$ is observed up to transverse momentum $\left(p_{\mathrm{T}}\right)$ of $60 \mathrm{GeV}$ in $\mathrm{Pb}+\mathrm{Pb}$ collisions. Compared to $\mathrm{Pb}+\mathrm{Pb}$ collisions, the $\mathrm{Xe}+\mathrm{Xe}$ $v_{2}$ is larger in the most central collisions. This can be attributed to larger fluctuations in $\mathrm{Xe}+\mathrm{Xe}$ and a possible deformation of the $\mathrm{Xe}$ nucleus, which leads to larger eccentricity in $\mathrm{Xe}+\mathrm{Xe}$ than in $\mathrm{Pb}+\mathrm{Pb}$. For mid-central and peripheral events, the $v_{2}$ in $\mathrm{Xe}+\mathrm{Xe}$ becomes smaller than in $\mathrm{Pb}+\mathrm{Pb}$, indicating the increased role of viscous effects. These trends are also observed for the higher-order harmonics and were predicted qualitatively by relativistic viscous hydrodynamics.

Two types of scaling behavior are observed for flow both in $\mathrm{Pb}+\mathrm{Pb}$ and $\mathrm{Xe}+\mathrm{Xe}$ collisions. The first scaling is that the $v_{n}$ for fixed $n$ have the same shape as a function of $p_{\mathrm{T}}$ across different centralities, up to an overall normalization and scaling along the $p_{\mathrm{T}}$ axis [4]. The second scaling is that the ratio $v_{n} / v_{m}^{n / m}$ for two harmonics of order $m$ and $n$ is independent of $p_{\mathrm{T}}$ in a given centrality interval [5,6]. Neither scaling is understood quantitatively. Besides a similarity captured by the scaling behavior there are also differences pointing to a role of fluctuations. One of them is the ordering of flow coefficients in ultra-central $(0-5 \%)$ collisions: $v_{3}>v_{4}>v_{2} \sim v_{5}$ for $p_{\mathrm{T}}$ above $1 \mathrm{GeV}$. Another is a weak centrality dependence of higher-order flow harmonics ( $v_{n}$ with $n \geq 3$ ). To better quantify the role of fluctuations, longitudinal flow decorrelations were measured in $\mathrm{Pb}+\mathrm{Pb}$ and $\mathrm{Xe}+\mathrm{Xe}$ collisions [7]. The decorrelation signal increases approximately linearly as a function of the $\eta$ separation between the two particles. The decorrelation was seen to be larger (smaller) for $v_{2}\left(v_{3}\right)$ in $\mathrm{Xe}+\mathrm{Xe}$ compared to $\mathrm{Pb}+\mathrm{Pb}$. While hydrodynamic models are found to describe the ratios of inclusive $v_{n}$ between $\mathrm{Xe}+\mathrm{Xe}$ and $\mathrm{Pb}+\mathrm{Pb}$, they fail to describe many of the magnitudes and trends in the longitudinal decorrelations between $\mathrm{Xe}+\mathrm{Xe}$ and $\mathrm{Pb}+\mathrm{Pb}$. This suggests that models tuned to describe the transverse dynamics may not necessarily describe the longitudinal structure of the initial state geometry. The new measurements may be used to improve the three-dimensional initial conditions for hydrodynamic models.

Event-by-event fluctuations of flow and correlations between flow harmonics were further studied in $\mathrm{Pb}+\mathrm{Pb}$ collisions [9] and in $13 \mathrm{TeV} p p, 5.02 \mathrm{TeV} p+\mathrm{Pb}$, and $2.76 \mathrm{TeV} \mathrm{Pb}+\mathrm{Pb}$ collisions [10] using symmetric and asymmetric subevent cumulants. These measurements revealed e.g. signs of significant nonGaussian fluctuations in $v_{2}$ and $v_{3}$ in ultra-central $\mathrm{Pb}+\mathrm{Pb}$ collisions, or a negative correlation between $v_{2}$ and $v_{3}$ and a positive correlation between $v_{2}$ and $v_{4}$ which is present for all studied collision systems. While the correlations among flow magnitudes are universal across collision systems, their magnitudes depend on the event multiplicity and the choice of the $p_{\mathrm{T}}$ range and the collision system which provides a clear 

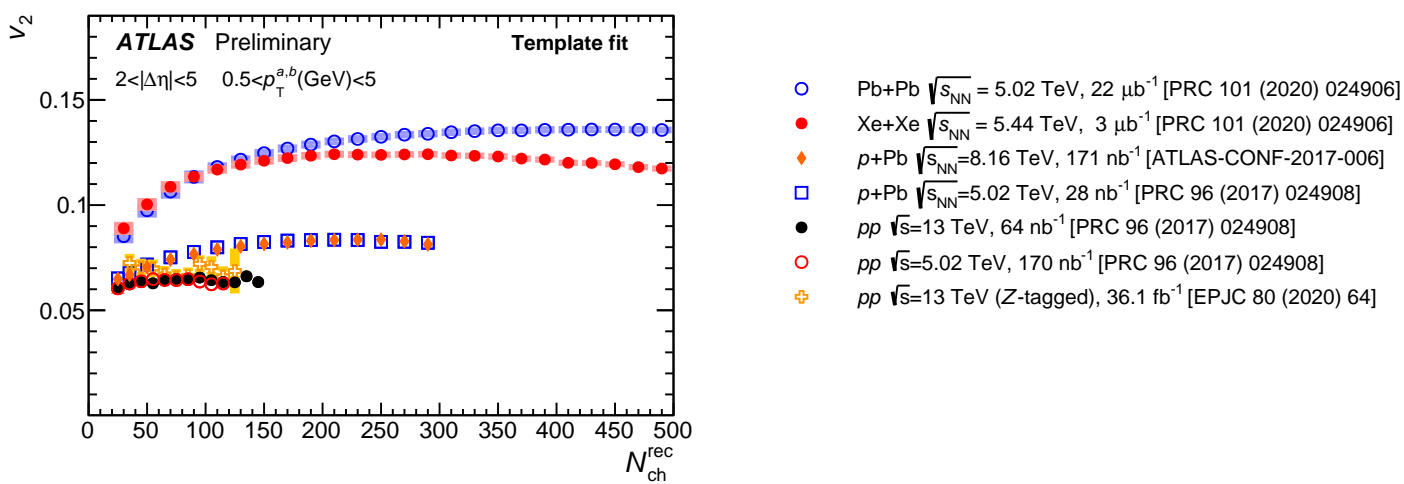

Fig. 1. Comparisons of the $v_{2}$ measured in different collision systems and collision energies as a function of the measured multiplicity, $N_{\mathrm{ch}}^{\mathrm{rec}}$. The $v_{2}$ values are obtained with the template-fit method. For the $\mathrm{Pb}+\mathrm{Pb}$ and $\mathrm{Xe}+\mathrm{Xe}$ measurements, the peripheral reference in the template fits is constructed using $p p$ collisions at $5.02 \mathrm{TeV}$ that have $N_{\mathrm{ch}}^{\mathrm{rec}}<20$. For all other systems, the peripheral reference is constructed using events from the corresponding collision system that have $N_{\mathrm{ch}}^{\mathrm{rec}}<20$. The plots are for $0.5 \mathrm{GeV}<p_{\mathrm{T}}<5 \mathrm{GeV}$. The error bars and bands represent statistical and systematic uncertainties, respectively, and are typically too small to be observed. Results taken from Ref. [8].

discriminative power for modeling the origin of flow in small collision systems.

The origin of flow in small collision systems may be better understood also using results from several other measurements. This first one is the measurement of the correlation between the flow harmonics $\left(v_{2}-v_{4}\right)$ and the mean $p_{\mathrm{T}}$ in $p+\mathrm{Pb}$ and $\mathrm{Pb}+\mathrm{Pb}$ collisions [11]. The magnitude of this correlation is quantified by modified Pearson correlation coefficient. A strong positive correlation is observed between $v_{2}$ and mean $p_{\mathrm{T}}$ in mid-central and central collisions while an anticorrelation is seen for peripheral events. An anticorrelation is observed also for $p+\mathrm{Pb}$ collisions, but exhibiting different trends compared to peripheral $\mathrm{Pb}+\mathrm{Pb}(\mathrm{a}$ growth of the correlation with number of charged particles in the event $\left(N_{\mathrm{ch}}\right)$ is seen in $\mathrm{Pb}+\mathrm{Pb}$, but no $N_{\mathrm{ch}}$ dependence is seen in $p+\mathrm{Pb})$. One of the clear next steps to disentangle the origin of these correlations is to measure these correlations in $p p$ collisions.

The second measurement is the measurement of elliptic flow of muons from the decay of charm and bottom hadrons in $13 \mathrm{TeV} p p$ collisions [12]. A significant non-zero $v_{2}$ is observed for muons from charm decays, while the $v_{2}$ value for muons from bottom decays is observed to be consistent with zero. This indicates that the collectivity in small collision systems does not persist up to the scale of heavy quark masses. This measurement can be compared to the measurement of the same quantities in $\mathrm{Pb}+\mathrm{Pb}$ collisions [13]. In the case of $\mathrm{Pb}+\mathrm{Pb}$, the $v_{2}$ and $v_{3}$ for muons from bottom decays are seen to be non-zero (but significantly smaller than the $v_{2}$ and $v_{3}$ for charm muons). The $\mathrm{Pb}+\mathrm{Pb}$ results can significantly discriminate between models of heavy-quark energy loss and constrain heavy-quark transport coefficients in the QGP.

The third measurement to help understanding the flow in small systems is a measurement of $v_{2}$ in ultraperipheral $\mathrm{Pb}+\mathrm{Pb}$ collisions [14]. Significant $v_{2}$ values of $4-6 \%$ are observed in these photo-nuclear events. The $v_{2}$ values have a similar dependence on $p_{\mathrm{T}}$ and $N_{\mathrm{ch}}$ as in $13 \mathrm{TeV} p p$ and $5.02 \mathrm{TeV} p+\mathrm{Pb}$ collisions, but an overall smaller magnitude. The signal of $v_{2}$ in photo-nuclear events may be understood in the vectormeson dominance picture as arising from a $\rho+\mathrm{Pb}$ or $\omega+\mathrm{Pb}$ interaction at a lower energy than the equivalent nucleon-nucleon collision energy.

The last measurements to be mentioned in the context of small systems is the measurement of ridge in $Z$-triggered events in $13 \mathrm{TeV}$ and $8 \mathrm{TeV} p p$ collisions [15]. A significant pile-up contribution to the twoparticle correlations is subtracted using a new pile-up correction method. The magnitude of the observed $v_{2}$ as a function of multiplicity and $p_{\mathrm{T}}$ is found to be consistent with that observed in inclusive $p p$ collisions. This measurement demonstrates that in $p p$ collisions, the long-range correlation involving soft particles is not significantly altered by the presence of a hard-scattering process.

It is also important to study the high- $p_{\mathrm{T}}$ limit of flow in small collision system. Such a study was done in $8.16 \mathrm{TeV} p+\mathrm{Pb}$ collisions [16]. The measurement was performed using minimum-bias events and events 

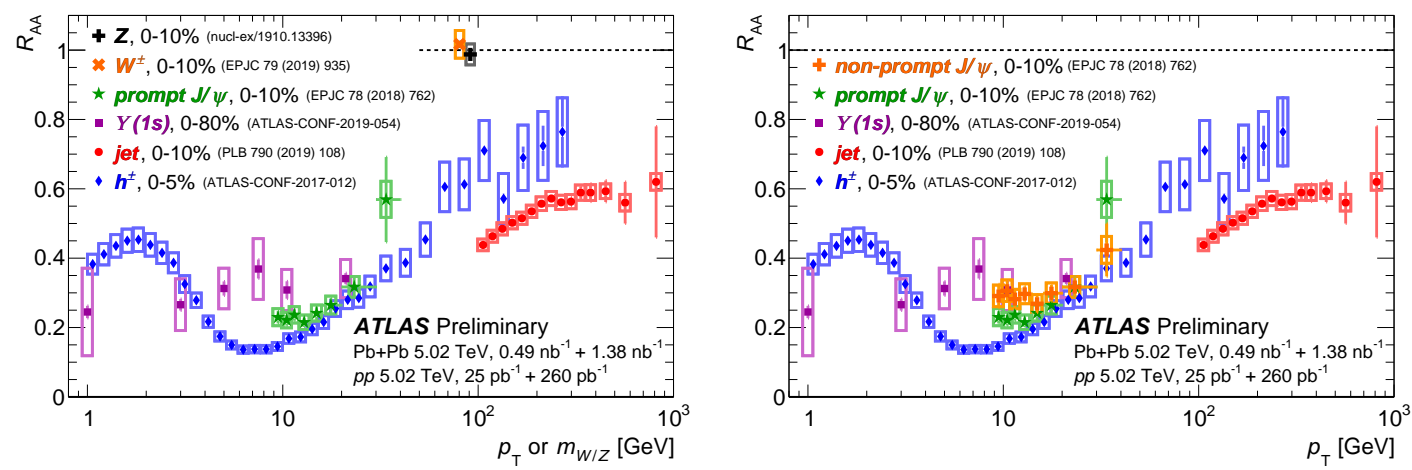

Fig. 2. Compilation of results for the nuclear modification factor $R_{\mathrm{AA}}$ vs. $p_{\mathrm{T}}$ or $m_{W / Z}$ in different channels from the Run $2 \mathrm{~Pb}+\mathrm{Pb}$ and $p p$ data. Left: Results for $Z$ bosons (black plus, plotted at the $Z$ boson mass), $W^{ \pm}$bosons (orange cross, plotted at the $W$ boson mass), anti- $k_{\mathrm{t}} R=0.4$ jets (red circles), charged hadrons (blue diamonds), prompt $J / \Psi$ 's (green stars), and $\Upsilon(1 S)$ particles (purple squares). Right: Results for anti- $k_{\mathrm{t}} R=0.4$ jets (red circles), charged hadrons (blue diamonds), prompt $J / \Psi$ 's (green stars), non-prompt $J / \Psi$ s (orange pluses), and $\Upsilon(1 S)$ particles (purple squares). In both panels, the statistical uncertainties are shown as vertical bars and the total systematic uncertainties, including $p p$ luminosity and $T_{\mathrm{AA}}$ uncertainties, are shown as boxes. Results taken from Ref. [8].

triggered by jets. The anisotropies for particles with $p_{\mathrm{T}}$ of $0.5-2 \mathrm{GeV}$ are consistent between minimum bias (MB) events and jet-triggered events and also consistent with trends predicted by relativistic hydrodynamics. In the $p_{\mathrm{T}}$ range of 2-9 GeV, the anisotropies are larger in $\mathrm{MB}$ events than in jet-triggered events which might be a result of changing admixture of particles from hard scattering and the underlying event. For $p_{\mathrm{T}}$ above $9 \mathrm{GeV}$, the $v_{n}$ results are again consistent between $\mathrm{MB}$ and jet-triggered events. The $v_{2}$ plateaus at a value of 0.025 up to a $p_{\mathrm{T}}$ of $50 \mathrm{GeV}$. This result cannot be explained in the theoretical context of jet quenching or "eremitic" expansion calculations while simultaneously describing the observed lack of suppression of high $-p_{\mathrm{T}}$ hadron and jet yields in $p+\mathrm{Pb}$ collisions.

Figure 1 presents a summary of selected results on flow in small systems.

\section{Penetrating probes of $\mathrm{QGP}$ in $\mathrm{Pb}+\mathrm{Pb}$ collisions}

Significant modifications of the internal structure of jets in central $\mathrm{Pb}+\mathrm{Pb}$ collisions compared to peripheral collisions or $p p$ collisions were established at ATLAS by measurements of inclusive jet fragmentation functions $[17,18,19]$. Yield of hadrons with $p_{\mathrm{T}}<4 \mathrm{GeV}$ is enhanced, yield of hadrons with $p_{\mathrm{T}}=4-20 \mathrm{GeV}$ is suppressed, and yield of hadrons with $p_{\mathrm{T}}>20 \mathrm{GeV}$ is seen to be enhanced in $\mathrm{Pb}+\mathrm{Pb}$ with respect to $p p$ collisions. ATLAS has now extended these measurements to study jet fragmentation in boson-triggered systems and to measure the radial dependence of fragmentation functions.

Fragmentation functions as a function of the radial distance from the jet axis $(r)$ were measured in $\mathrm{Pb}+\mathrm{Pb}$ and $p p$ collisions extending up to $r=0.8$. A broadening of jets for low- $p_{\mathrm{T}}$ particles $\left(p_{\mathrm{T}}<4 \mathrm{GeV}\right)$ and a narrowing of jets for high- $p_{\mathrm{T}}$ particles $\left(p_{\mathrm{T}}>4 \mathrm{GeV}\right)$ is seen, at the same time the core of the jet $(r<0.05)$ remains almost unmodified. This may help to qualitatively constrain the role of the wide-angle radiation, medium back-reaction to the propagating parton, or a role of the flavor of the initial parton.

Internal structure of jets was also measured for jets from $\gamma$-jet systems [20]. While in the $30-80 \%$ centrality bin the $\mathrm{Pb}+\mathrm{Pb}$-to- $\mathrm{pp}$ ratios of fragmentation functions are measured to be consistent between the inclusive and $\gamma$-tagged jets, a difference between the inclusive and $\gamma$-tagged jets is seen for the $0-$ $30 \%$ centrality bin. The minimum of the ratio is significantly shifted toward higher $p_{\mathrm{T}}$ of hadrons and the magnitude of the low- $p_{\mathrm{T}}$ enhancement is also larger. These fully particle-level corrected results along with results from measurements of photon-jet transverse momentum correlations [21] may allow further constraints to be put on the models of the energy loss.

It is not simple to extend the measurements of jet fragmentation with fully reconstructed jets to the kinematic region of jets with $p_{\mathrm{T}}$ of a few tens of $\mathrm{GeV}$ (e.g. due to inefficiencies which may be different 
for quenched and non-quenched jets). The alternative, allowing to go lower in $p_{\mathrm{T}}$, is a measurement of conditional yields of charged particles. The measurement of charged particle yields in $Z$-tagged events was performed in $\mathrm{Pb}+\mathrm{Pb}$ and $p p$ collisions [22]. The integrated yield was measured over $|\Delta \phi|>3 / 4 \pi$ from the $Z$ boson direction and the $\mathrm{Pb}+\mathrm{Pb}$-to- $p p$ ratio, $I_{\mathrm{AA}}$, of this yield was evaluated. The $I_{\mathrm{AA}}$ exhibits similar trends as those seen in fragmentation functions but with an overall larger amplitude of the modification which might be due to the fact that $I_{\mathrm{AA}}$ is a result of combination of the jet suppression with modified fragmentation. It might also reflect larger modifications of jets at lower $p_{\mathrm{T}}$.

To further improve on the understanding of the modifications of jet internal structure, ATLAS measured the nuclear modification factor, $R_{\mathrm{AA}}$, of jets for large-radius jets. The large-radius jets are reconstructed with the anti- $k_{\mathrm{t}}$ algorithm using a radius parameter of $R=1.0$, by re-clustering anti- $k_{\mathrm{t}} R=0.2$ jets with $p_{\mathrm{T}}>35 \mathrm{GeV}$ [23]. After applying a trimming procedure, the large-radius jet constituents are re-clustered using the $k_{\mathrm{t}}$ algorithm in order to obtain the splitting parameter, $\sqrt{d_{12}}$, which characterizes the transverse momentum scale for the hardest splitting in the jet. The inclusive jet $R_{\mathrm{AA}}$ is qualitatively similar to the previously measured $R_{\mathrm{AA}}$ of $R=0.4$ jets [24] confirming a presence of sizable jet quenching at a scale of $1 \mathrm{TeV}$. When measured as a function of $\sqrt{d_{12}}$, a significant evolution of $R_{\mathrm{AA}}$ is observed. The $R_{\mathrm{AA}}$ values for jets with small $\sqrt{d_{12}}$ (corresponding to jets with just one single subjet) were found to be significantly larger compared to the $R_{\mathrm{AA}}$ for other $\sqrt{d_{12}}$ values (that is those having a more complex substructure).

It is not only jets or inclusive charged particles which are measured to be strongly suppressed in $\mathrm{Pb}+\mathrm{Pb}$ collisions. Figure 2 presents a summary of selected suppression measurements for various final states. New results of the measurement of $\Upsilon$ production [25] quantify the $R_{\mathrm{AA}}$ of $\Upsilon(1 S), \Upsilon(2 S)$, and $\Upsilon(2 S+3 S)$ states. An expected ordering of the suppression reflecting the binding energy of $\Upsilon$ states is seen: $R_{\mathrm{AA}}[\Upsilon(1 S)]>$ $R_{\mathrm{AA}}[\Upsilon(2 S)]>R_{\mathrm{AA}}[\Upsilon(2 S+3 S)]$. No significant $p_{\mathrm{T}}$ and rapidity dependence of the suppression is seen. All the suppression measurements presented here should be checked against effects from the initial geometry of the collision and modifications of parton distribution functions in the environment of the nucleus. This may be done based on the measurements presented in the next section.

\section{Physics of initial state and nuclear geometry}

Electroweak bosons production in $\mathrm{Pb}+\mathrm{Pb}$ collisions may be considered to be standard candles for suppression measurements presented in the previous section. ATLAS have measured $Z$ and $W$ boson production $[26,27]$ in $\mathrm{Pb}+\mathrm{Pb}$ collisions in the electron and muon decay channels. The measurements in $\mathrm{Pb}+\mathrm{Pb}$ collisions are compared with those made in $p p$ collisions [28] and the $R_{\mathrm{AA}}$ is calculated. The results are then compared with predictions obtained at next-to-leading order (NLO) using nucleon and nuclear parton distribution functions (nPDFs). The $R_{\mathrm{AA}}$ of $Z$ is found to be consistent with unity for all centrality intervals. The nuclear modification factor measured as a function of rapidity agrees with unity and is consistent with an NLO QCD calculation including the isospin effect. The $R_{\mathrm{AA}}$ of $\mathrm{W}$ bosons is also found to be consistent with theoretical predictions. No dependence of normalized $\mathrm{W}$ yields on centrality and a good agreement with predictions are observed for mid-central and central collisions. For peripheral collisions, the data agree with predictions within $1.7(0.9)$ standard deviations for $\mathrm{W}^{-}\left(\mathrm{W}^{+}\right)$bosons. The trend observed in the $R_{\mathrm{AA}}$ for peripheral collisions goes in the opposite direction to predictions including the centrality bias evaluated with the HG-PYTHIA model [29] for charged-hadron production measured previously by ALICE [30]. New precision measurements of $W$ and $Z$ bosons production in $\mathrm{Pb}+\mathrm{Pb}$ collisions have confirmed the expected smallness of nPDF effects in the studied kinematic region and provide new constraints on these effects.

Initial state effects are further studied in two $p+\mathrm{Pb}$ measurements. The first one is the measurement of inclusive production of prompt, isolated photons in $p+\mathrm{Pb}$ collisions at $8.16 \mathrm{TeV}$ [31]. A measurement of the nuclear modification factor is done for photons with transverse energies in the region of $25-550 \mathrm{GeV}$ using a NLO pQCD-based extrapolation of previously published $p p$ data at $\sqrt{s}=8 \mathrm{TeV}$. The data are compatible with the expectation that the parton distribution functions are modestly modified in nuclei in this kinematic region and may help to place an upper limit on the possible amount of energy lost by partons in the initial stages of nuclear collisions.

The second $p+\mathrm{Pb}$ measurement is the measurement of forward-forward and forward-central dijet azimuthal angular correlations and conditional yields in $p p$ and $p+\mathrm{Pb}$ collisions [32] (the forward rapidity 
interval is $2.7<y<4.0$ ). These measurements probe the nuclear gluon density in regions of small parton momentum fractions $(x)$ where gluon saturation may play a role. No significant broadening of azimuthal angular correlations is observed for forward-forward or forward-central dijets in $p+\mathrm{Pb}$ compared to $p p$ collisions. For forward-forward jet pairs in the proton-going direction, the ratio of conditional yields in $p+\mathrm{Pb}$ collisions to those in $p p$ collisions is suppressed by approximately $20 \%$, with no significant dependence on the $p_{\mathrm{T}}$ of the dijet system. No modification of conditional yields is observed for forward-central dijets. The suppression seen in the data may be used to constrain possible nuclear effects including saturation.

\section{Ultra-peripheral collisions}

Boosted proton and nuclei are sources of significant electromagnetic (EM) fields. These EM fields can be treated as a beam of quasi-real photons with a small virtuality of $Q^{2}<1 / R^{2}$, where $R$ is the radius of the charge distribution $\left(Q^{2}<10^{-3} \mathrm{GeV}^{2}\right.$ for lead ions). In ultra-peripheral collision (UPC) events, with impact parameters larger than twice the radius of the nuclei, the strong interaction does not play a role and the only interaction comes from EM fields.

Since the photon flux associated with each nucleus scales as $Z^{2}$, the cross section for photon induced processes is extremely enhanced as compared to $p p$ collisions. One of interesting processes that may be studied in this configuration is the light-by-light scattering. This first strong evidence for that process was reported by ATLAS in 2017 [33] with a significance of $4.4 \sigma$. New measurement of the light-by-light scattering using $2018 \mathrm{~Pb}+\mathrm{Pb}$ data [34] brought an observation of this process at the level of $8.2 \sigma$ and also lowered the kinematic thresholds. In the new measurement, candidates are selected in events with two photons produced exclusively, each with transverse energy, $E_{\mathrm{T}}>3 \mathrm{GeV}$, diphoton invariant mass, $m_{\gamma \gamma}>6 \mathrm{GeV}$, and small diphoton transverse momentum and acoplanarity. After applying all selection criteria, 59 candidate events are observed for a background expectation of $12 \pm 3$ events. Besides being a precision test of QED, the light-by-light scattering may also put limits on production of particles beyond the Standard Model such as axion-like particles, magnetic monopoles, or vector-like fermions [35].

The EM fields are naturally also present in inelastic heavy-ion collisions. Production of muons from the process $\gamma \gamma \rightarrow \mu^{+} \mu^{-}$may bring a new way to study EM degrees of freedom of QGP. This "non-UPC" dimuon production was studied first using $2015 \mathrm{~Pb}+\mathrm{Pb}$ data [36] and later using 2015+2018 $\mathrm{Pb}+\mathrm{Pb}$ data [37]. In peripheral collisions, the muons exhibit a strong, expected back-to-back correlation. In central collisions, the angular correlations are observed to be broadened significantly. This is quantified in the original measurement [36] by measuring the acoplanarity distributions (acoplanarity $\alpha=1-\Delta \phi / \pi$, where $\Delta \phi$ is the azimuthal angle between muons). With the improved statistical precision of the new measurement [37], an additional depletion is observed in the acoplanarity distributions near $\alpha=0$. This was further quantified by measuring the $k_{\perp}=\bar{p}_{T}|\pi-\Delta \phi|$ (where $\bar{p}_{T}$ is the average transverse momentum of muons). While the $\alpha$ distributions are observed to vary strongly with $\bar{p}_{T}$, the distributions of $k_{\perp}$ are observed to be approximately independent of $\bar{p}_{T}$ as would be expected if the modification of the distributions results from a mechanism that imparts a momentum to one or both of the muons in the final state or to the photons in the initial state. The $k_{\perp}$ distributions show a centrality-dependent shift of the most-probable $k_{\perp}$ value away from $k_{\perp}=0$. In the $0-5 \%$ most central collisions the peak in the $k_{\perp}$ distribution is shifted by $36 \pm 1 \mathrm{MeV}$.

\section{Conclusions}

The ATLAS experiment at the LHC has undertaken an extensive heavy-ion physics program. In this short report we have summarized the latest results on the collectivity in $p p, p+\mathrm{Pb}$ and nucleus-nucleus collisions, results on penetrating probes of QGP in $\mathrm{Pb}+\mathrm{Pb}$ collisions, results on physics of initial state and nuclear geometry, and results on the electromagentic production in ultra-peripheral collisions. New results by ATLAS should help to answer many open questions in the field. They also open doors to the Run 3 heavy-ion physics summarized in Ref. [38]. 


\section{Acknowledgment}

This work was supported by Grant Agency of the Czech Republic under grant 18-12859Y, by the Ministry of Education, Youth and Sports of the Czech Republic under grant LTT 17018, and by Charles University grant UNCE/SCI/013.

Copyright 2020 CERN for the benefit of the ATLAS Collaboration. CC-BY-NC-ND-4.0 license.

\section{References}

[1] ATLAS Collaboration, The ATLAS Experiment at the CERN Large Hadron Collider, JINST 3 (2008) S08003. doi:10.1088/17480221/3/08/S08003.

[2] ATLAS Collaboration, Measurement of the azimuthal anisotropy of charged particles produced in $\sqrt{s_{\mathrm{NN}}}=5.02 \mathrm{TeV} \mathrm{Pb}+\mathrm{Pb}$ collisions with the ATLAS detector, Eur. Phys. J. C78 (12) (2018) 997. arXiv:1808.03951, doi:10.1140/epjc/s10052-018-6468-7.

[3] ATLAS Collaboration, Measurement of the azimuthal anisotropy of charged-particle production in Xe+Xe collisions at $\sqrt{s_{\mathrm{NN}}}=$ 5.44 TeV with the ATLAS detectorarXiv:1911.04812.

[4] A. Collaboration, Measurement of long-range pseudorapidity correlations and azimuthal harmonics in $\sqrt{s_{N N}}=$ 5.02 TeV proton-lead collisions with the ATLAS detector, Phys. Rev. C90 (4) (2014) 044906 arXiv:1409.1792, doi:10.1103/PhysRevC.90.044906.

[5] S. Collaboration, Azimuthal anisotropy at RHIC: The First and fourth harmonics, Phys. Rev. Lett. 92 (2004) 062301. arXiv:nuclex/0310029, doi:10.1103/PhysRevLett.92.062301.

[6] A. Collaboration, Measurement of the azimuthal anisotropy for charged particle production in $\sqrt{s_{N N}}=2.76 \mathrm{TeV}$ lead-lead collisions with the ATLAS detector, Phys. Rev. C86 (2012) 014907. arXiv:1203.3087, doi:10.1103/PhysRevC.86.014907.

[7] ATLAS Collaboration, Longitudinal flow decorrelations in $\mathrm{Xe}+\mathrm{Xe}$ collisions at $\sqrt{s_{\mathrm{NN}}}=5.44 \mathrm{TeV}$ with the ATLAS detector, ATLAS-CONF-2019-055.

[8] ATLAS Collaboration, Compilation plots of Run 2 Heavy Ions results for Quark Matter 2019, ATLAS-PUB-2020-003.

[9] ATLAS Collaboration, Fluctuations of anisotropic flow in $\mathrm{Pb}+\mathrm{Pb}$ collisions at $\sqrt{s_{\mathrm{NN}}}=5.02 \mathrm{TeV}$ with the ATLAS detector, Submitted to: JHEParXiv:1904.04808.

[10] ATLAS Collaboration, Correlated long-range mixed-harmonic fluctuations measured in $p p, p+\mathrm{Pb}$ and low-multiplicity $\mathrm{Pb}+\mathrm{Pb}$ collisions with the ATLAS detector, Phys. Lett. B789 (2019) 444-471. arXiv:1807.02012, doi:10.1016/j.physletb.2018.11.065.

[11] ATLAS Collaboration, Measurement of flow harmonics correlations with mean transverse momentum in lead-lead and protonlead collisions at $\sqrt{s_{N N}}=5.02 \mathrm{TeV}$ with the ATLAS detectorarXiv:1907.05176.

[12] ATLAS Collaboration, Measurement of azimuthal anisotropy of muons from charm and bottom hadrons in $p p$ collisions at $\sqrt{s}=13 \mathrm{TeV}$ with the ATLAS detectorarXiv:1909.01650.

[13] ATLAS Collaboration, Measurement of azimuthal anisotropy of muons from charm and bottom hadrons in $\mathrm{Pb}+\mathrm{Pb}$ collisions at $\sqrt{s_{\mathrm{NN}}}=5.02 \mathrm{TeV}$ with the ATLAS detector, ATLAS-CONF-2019-053.

[14] ATLAS Collaboration, Two-particle azimuthal correlations in photo-nuclear ultra-peripheral $\mathrm{Pb}+\mathrm{Pb}$ collisions at $5.02 \mathrm{TeV}$ with ATLAS, ATLAS-CONF-2019-022.

[15] ATLAS Collaboration, Measurement of long-range two-particle azimuthal correlations in $Z$-boson tagged $p p$ collisions at $\sqrt{s}=8$ and 13 TeVarXiv:1906.08290.

[16] ATLAS Collaboration, Transverse momentum and process dependent azimuthal anisotropies in $\sqrt{s_{\mathrm{NN}}}=8.16 \mathrm{TeV} p+\mathrm{Pb}$ collisions with the ATLAS detectorarXiv:1910.13978.

[17] ATLAS Collaboration, Measurement of inclusive jet charged-particle fragmentation functions in $\mathrm{Pb}+\mathrm{Pb}$ collisions at $\sqrt{s_{N N}}=2.76$ TeV with the ATLAS detector, Phys. Lett. B739 (2014) 320-342. arXiv:1406.2979, doi:10.1016/j.physletb.2014.10.065.

[18] ATLAS Collaboration, Measurement of jet fragmentation in $\mathrm{Pb}+\mathrm{Pb}$ and $p p$ collisions at $\sqrt{s_{\mathrm{NN}}}=2.76 \mathrm{TeV}$ with the ATLAS detector at the LHC, Eur. Phys. J. C77 (6) (2017) 379. arXiv:1702.00674, doi:10.1140/epjc/s10052-017-4915-5.

[19] ATLAS Collaboration, Measurement of jet fragmentation in $\mathrm{Pb}+\mathrm{Pb}$ and $p p$ collisions at $\sqrt{s_{N N}}=5.02 \mathrm{TeV}$ with the ATLAS detector, Phys. Rev. C98 (2) (2018) 024908. arXiv:1805.05424, doi:10.1103/PhysRevC.98.024908.

[20] ATLAS Collaboration, Comparison of Fragmentation Functions for Jets Dominated by Light Quarks and Gluons from $p p$ and $\mathrm{Pb}+\mathrm{Pb}$ Collisions in ATLAS, Phys. Rev. Lett. 123 (4) (2019) 042001. arXiv:1902.10007, doi:10.1103/PhysRevLett.123.042001.

[21] ATLAS Collaboration, Measurement of photon-jet transverse momentum correlations in $5.02 \mathrm{TeV} \mathrm{Pb}+\mathrm{Pb}$ and $p p$ collisions with ATLAS, Phys. Lett. B789 (2019) 167-190. arXiv:1809.07280, doi:10.1016/j.physletb.2018.12.023.

[22] ATLAS Collaboration, Measurement of $Z$-tagged charged-particle yields in $5.02 \mathrm{TeV} \mathrm{Pb}+\mathrm{Pb}$ and $p p$ collisions with the ATLAS detector, ATLAS-CONF-2019-052.

[23] ATLAS Collaboration, Measurement of suppression of large-radius jets and its dependence on substructure in $\mathrm{Pb}+\mathrm{Pb}$ at $5.02 \mathrm{TeV}$ by ATLAS detector, ATLAS-CONF-2019-056.

[24] ATLAS Collaboration, Measurement of the nuclear modification factor for inclusive jets in $\mathrm{Pb}+\mathrm{Pb}$ collisions at $\sqrt{s_{\mathrm{NN}}}=5.02$ TeV with the ATLAS detector, Phys. Lett. B790 (2019) 108-128. arXiv:1805.05635, doi:10.1016/j.physletb.2018.10.076.

[25] ATLAS Collaboration, Production of $\Upsilon(\mathrm{nS})$ mesons in $\mathrm{Pb}+\mathrm{Pb}$ and $p p$ collisions at 5.02 TeV with ATLAS, ATLAS-CONF-2019054.

[26] ATLAS Collaboration, $Z$ boson production in $\mathrm{Pb}+\mathrm{Pb}$ collisions at $\sqrt{s_{\mathrm{NN}}}=5.02 \mathrm{TeV}$ measured by the ATLAS experimentarXiv:1910.13396. 
[27] ATLAS Collaboration, Measurement of $W^{ \pm}$boson production in $\mathrm{Pb}+\mathrm{Pb}$ collisions at $\sqrt{s_{\mathrm{NN}}}=5.02$ TeV with the ATLAS detector, Eur. Phys. J. C79 (11) (2019) 935. arXiv:1907.10414, doi:10.1140/epjc/s10052-019-7439-3.

[28] ATLAS Collaboration, Measurements of $W$ and $Z$ boson production in $p p$ collisions at $\sqrt{s}=5.02$ TeV with the ATLAS detector, Eur. Phys. J. C79 (2) (2019) 128, [Erratum: Eur. Phys. J.C79,no.5,374(2019)]. arXiv:1810.08424, doi:10.1140/epjc/s10052-0196870-9, 10.1140/epjc/s10052-019-6622-x.

[29] C. Loizides, A. Morsch, Absence of jet quenching in peripheral nucleus-nucleus collisions, Phys. Lett. B773 (2017) $408-411$. arXiv:1705.08856, doi:10.1016/j.physletb.2017.09.002.

[30] ALICE Collaboration, Analysis of the apparent nuclear modification in peripheral Pb-Pb collisions at 5.02 TeV, Phys. Lett. B793 (2019) 420-432. arXiv:1805.05212, doi:10.1016/j.physletb.2019.04.047.

[31] ATLAS Collaboration, Measurement of prompt photon production in $\sqrt{s_{\mathrm{NN}}}=8.16 \mathrm{TeV} p+\mathrm{Pb}$ collisions with ATLAS, Phys. Lett. B796 (2019) 230-252. arXiv:1903.02209, doi:10.1016/j.physletb.2019.07.031.

[32] ATLAS Collaboration, Dijet azimuthal correlations and conditional yields in pp and $\mathrm{p}+\mathrm{Pb}$ collisions at $\mathrm{sNN}=5.02 \mathrm{TeV}$ with the ATLAS detector, Phys. Rev. C100 (3) (2019) 034903. arXiv:1901.10440, doi:10.1103/PhysRevC.100.034903.

[33] ATLAS Collaboration, Evidence for light-by-light scattering in heavy-ion collisions with the ATLAS detector at the LHC, Nature Phys. 13 (9) (2017) 852-858. arXiv:1702.01625, doi:10.1038/nphys4208.

[34] ATLAS Collaboration, Observation of light-by-light scattering in ultraperipheral $\mathrm{Pb}+\mathrm{Pb}$ collisions with the ATLAS detector, Phys. Rev. Lett. 123 (5) (2019) 052001. arXiv:1904.03536, doi:10.1103/PhysRevLett.123.052001.

[35] ATLAS Collaboration, Prospects for Measurements of Photon-Induced Processes in Ultra-Peripheral Collisions of Heavy Ions with the ATLAS Detector in the LHC Runs 3 and 4, ATL-PHYS-PUB-2018-018.

[36] ATLAS Collaboration, Observation of centrality-dependent acoplanarity for muon pairs produced via two-photon scattering in $\mathrm{Pb}+\mathrm{Pb}$ collisions at $\sqrt{s_{\mathrm{NN}}}=5.02 \mathrm{TeV}$ with the ATLAS detector, Phys. Rev. Lett. 121 (21) (2018) 212301. arXiv:1806.08708, doi:10.1103/PhysRevLett.121.212301.

[37] ATLAS Collaboration, Measurement of non-exclusive dimuon pairs produced via $\gamma \gamma$ scattering in $\mathrm{Pb}+\mathrm{Pb}$ collisions at $\sqrt{s_{\mathrm{NN}}}=$ $5.02 \mathrm{TeV}$ with the ATLAS detector, ATLAS-CONF-2019-051.

[38] Z. Citron, et al., Report from Working Group 5arXiv:1812.06772, doi:10.23731/CYRM-2019-007.1159. 